\title{
Magnetic Localized Surface Plasmons
}

\author{
Paloma A. Huidobro, ${ }^{1}$ Xiaopeng Shen, ${ }^{2}$ J. Cuerda, ${ }^{1}$ Esteban Moreno, ${ }^{1}$ L. Martin-Moreno, ${ }^{3}$ \\ F. J. Garcia-Vidal, ${ }^{1, *}$ Tie Jun Cui, ${ }^{2, \dagger}$ and J. B. Pendry ${ }^{4}$ \\ ${ }^{1}$ Departamento de Fisica Teorica de la Materia Condensada and Condensed Matter Physics \\ Center (IFIMAC), Universidad Autonoma de Madrid, 28049 Madrid, Spain \\ ${ }^{2}$ State Key Laboratory of Millimetre Waves, School of Information Science and Engineering, \\ Southeast University, Nanjing 210096, China \\ ${ }^{3}$ Instituto de Ciencia de Materiales de Aragon and Departamento de Fisica de la Materia Condensada, \\ CSIC-Universidad de Zaragoza, E-50009 Zaragoza, Spain \\ ${ }^{4}$ Department of Physics, The Blackett Laboratory, Imperial College London, \\ London SW7 2AZ, United Kingdom
}

(Received 23 October 2013; revised manuscript received 3 February 2014; published 3 April 2014)

\begin{abstract}
Here, we introduce the concept of magnetic localized surface plasmons (LSPs), magnetic dipole modes that are supported by cylindrical metal structures corrugated by very long, curved grooves. The resonance wavelength is dictated by the length of the grooves, allowing us to tune it to values much larger than the size of the particle. Moreover, magnetic LSPs also exist for extremely thin metal disks and, therefore, they could be used to devise metasurfaces with magnetic functionalities. Experimental evidence of the existence of these magnetic LSPs in the microwave regime is also presented, although the concept is very general and could be applied to terahertz or infrared frequencies.
\end{abstract}

DOI: 10.1103/PhysRevX.4.021003

Surface plasmons are the subject of intense interest at optical frequencies [1-3]. Their ability to capture photons from the far field into short-wavelength surface excitations on metal surfaces offers the potential for controlling light on a scale much less than the wavelength. The fact that subwavelength nanoparticles are able to focus light into areas of the order of a few square nanometers enables huge concentrations of optical energy, greatly enhancing the sensitivity of spectroscopic detection of biochemical species [4]. Until recently, plasmonics studies have been confined to the higher frequencies: the UV, visible, and near infrared. At lower frequencies (terahertz regions or rf frequencies), plasmons do not feature in electromagnetic properties. However, a highly conducting surface can be structured so as to support surface waves $[5,6]$ that in many respects mimic, or "spoof," real surface plasmons [7]. These spoof surface plasmons open up the terahertz or rf bands to plasmonic studies [8-14].

Here in this work, we go one step further. Conventionally, surface plasmons are electric excitations consisting of oscillations in the density of metallic

\footnotetext{
*Corresponding author. fj.garcia@uam.es

Corresponding author. tjcui@seu.edu.cn

Published by the American Physical Society under the terms of the Creative Commons Attribution 3.0 License. Further distribution of this work must maintain attribution to the author(s) and the published article's title, journal citation, and DOI.
}

Subject Areas: Metamaterials, Optics, Plasmonics

conduction electrons. In a metamaterial world, there is also the possibility of creating magnetic excitations [15-22], such as magnetic surface plasmons propagating along the planar surface of a metamaterial with negative permittivity [23]. In contrast, the subject of the present paper will be spoof magnetic localized surface plasmons. We explore both theoretically and experimentally cylindrical structures with external dimensions much less than the free-space wavelength and show that they support magnetic as well as electrical surface modes. These structures are the lowfrequency analogues of the subwavelength nanoparticles studied at optical frequencies and offer the same potential for diverse applications.

Let us start by revisiting the two-dimensional (2D) structure shown in Fig. 1(a), which has already been studied by some of the authors of this work [24] and which has also been considered for waveguiding purposes in the earlier literature; see, e.g., Ref. [25]. It consists of an infinitely long metallic cylinder of radius $R$ corrugated with a periodic array (period $d=2 \pi R / N$ ) of $N$ grooves of parallel walls, with depth $h=R-r$ and width $a$. This 2D geometry supports similar electromagnetic (EM) resonances as its three-dimensional (3D) counterpart (disks) and, importantly, allows for an analytical treatment of its EM response. For proof-of-principle purposes, we take the cylinder as made of a perfect electrical conductor (PEC), which is a very good approximation for sufficiently low frequencies. The grooves are filled with a dielectric material of refractive index $n_{g}$. In this way, the resonant wavelengths $\lambda_{\text {res }}$ can be made much larger than the size of 
(a)

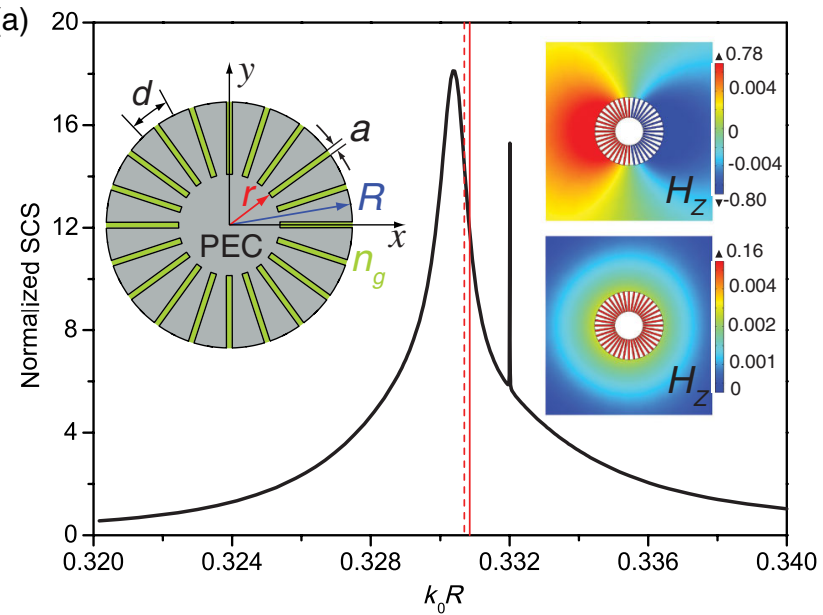

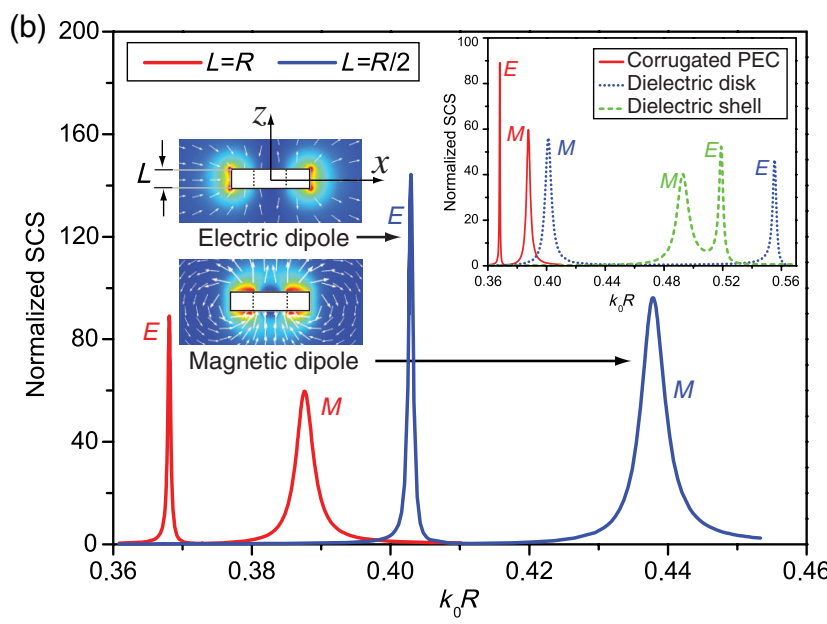

FIG. 1. Magnetic LSPs in subwavelength PEC disks corrugated with dielectric grooves. (a) SCS for a 2D structure with $r=0.4 R$, $N=40, a / d=0.3$, and $n_{g}=8$. The low-energy peak $\left(k_{0} R=0.33\right)$ reveals two resonances: the electrical and magnetic dipoles, whose magnetic field amplitudes $\left(H_{z}\right)$ are plotted in the upper and lower right insets, respectively. The solid (dashed) vertical lines show the position of the electric (magnetic) resonance according to the analytical model. (b) Structures of finite thickness. SCS for corrugated disks of thickness $L=R$ (red line) and $L=R / 2$ (blue line), with the same parameters as in (a). Top (and bottom) left insets: Near-field distribution of the electric (and magnetic) dipole resonances for a disk of thickness $L=R / 2$. The arrows show the electric (magnetic) field lines, and the color map shows the norm of the electric (magnetic) field. Upper right inset: SCS for a corrugated disk of $L=R$ (solid red line), a dielectric disk of the same size and $n_{g}=8$ (dotted blue line), and a PEC core of radius $r$ with a dielectric shell of radius $R$ and $n_{g}=8$ (dashed green line).

the object and we can operate in the very deep subwavelength regime $R \ll \lambda_{\text {res. }}$. The EM response of this structure is analyzed by means of the scattering cross section (SCS), which is numerically computed via a finite element method [26]. In order to calculate the SCS, we consider (here and in the following) an incoming TM-polarized plane wave (magnetic field $\vec{H}$ pointing along the $z$ direction) propagating along the $x$ axis and with wave number $k_{0}=\omega / c$. We normalize the SCS by the geometrical cross section of the structure $2 R$. Since we are considering PECs, the resonant wavelengths scale with $R$, which is taken as unit length, and the frequency can be expressed in terms of the dimensionless quantity $k_{0} R$. The SCS spectrum for a representative textured cylinder with parameters $r=0.4 R$, $N=40$, and $a / d=0.3$ is shown in Fig. 1(a). This spectrum closely resembles what is observed for very subwavelength metal particles at optical frequencies. This analogy suggests that the first resonance peak is due to the electrical dipole resonance, while the high-frequency narrow peak is associated with the electrical quadrupole. The assignment of this high-frequency peak is correct, and we will not discuss it in this work. However, the eigenmode calculation of the structure reveals the unexpected (and previously missed) point that the low-frequency resonance does not have a pure electric dipole character, having instead contributions of two different EM modes with very similar eigenfrequencies. One of them has an electrical dipole character [see the upper right inset of Fig. 1(a)], whereas the field pattern associated with the other one is independent of the azimuthal angle, as corresponds to a magnetic dipole pointing along the $z$ axis [lower right inset of Fig. 1(a)]. This behavior is at odds with what happens in standard plasmonics, in which the magnetic dipole resonance is extremely weak and does not show up in the SCS of the subwavelength metal particle [27].

An analytical model based on the modal expansion technique gives a deep physical insight on the EM response of the 2D corrugated cylinder. This model is the translation of previous approaches devoted to analyzing the surface EM modes supported by a corrugated plane [28] in the case of a PEC cylinder with longitudinal corrugations. This modal expansion relies on the matching of the EM modes outside the cylinder (radial coordinate $\rho>R$ ) and inside the grooves $(r<\rho<R)$ by means of the appropriate boundary conditions at $\rho=R$. Expanding the EM fields in the outer region in terms of the Hankel function of the first kind $H_{n}^{(1)}$ and considering that, for subwavelength particles, the field within the groove is given only by the fundamental waveguide mode, we arrive at the following transcendental equation for the complex resonance frequency of the EM mode with azimuthal number $n$ :

$$
S_{n}^{2} \frac{H_{n}^{(1)}\left(k_{0} R\right)}{H_{n}^{(1) \prime}\left(k_{0} R\right)} \tan \left(k_{0} n_{g} h\right)=-n_{g},
$$

where $\quad S_{n}=\sqrt{a / d} \operatorname{sinc}[n \pi a /(N d)] \quad$ and $\quad H_{n}^{(1) \prime}(x) \equiv$ $d H_{n}^{(1)}(x) / d x$. Physically, the picture that emerges from 
this model is that of surface EM modes running around the cylinder surface, with each resonance appearing when an integer number of modal wavelengths fits into the perimeter. In Eq. (1), the tangent function accounts for the fact that a guided mode is traveling down the groove (of depth $h$ and refractive index $n_{g}$ ) and bouncing at its bottom. Within the language of antenna engineering, the term $S_{n}^{2} \tan \left(k_{0} n_{g} h\right)$ could be regarded as the normalized surface impedance of the structure [28]. In the subwavelength limit, Eq. (1) predicts that the mode for $n=0$ (i.e., azimuthally independent) is very close in frequencies to the mode for $n=1$, as shown by the vertical lines in Fig. 1(a). This analytical model can also be applied to calculate the associated SCS and the relative contribution of the $n=0,1$ modes (see Fig. S1 of the Supplemental Material [29]). Despite the few ingredients it includes, this simple modeling shows a very good agreement with the numerics, illustrates the geometrical origin of the modes, and justifies their naming: magnetic dipole localized surface plasmon (LSP) for the $n=0$ mode and electrical dipole LSP for the $n=1$ mode.

Going from the infinitely long, textured cylinder (2D) to a 3D corrugated disk of finite thickness $L$ has a fundamental consequence. Not only do the resonances shift when the size of the particle is reduced, as usual in particle plasmonics, but the magnetic and electric LSPs split in such a way that the former shifts to higher frequencies than the latter. The SCSs for two disks of different $L$ corrugated with grooves filled with a dielectric material are shown in Fig. 1(b). As in the 2D case, we have considered an incident wave polarized with the magnetic field pointing in the $z$ direction and propagating along the $x$ axis. The SCS is normalized by the geometrical cross section, which in this case is $2 R L$. Two distinct peaks can be seen in the SCS plot for disks of finite thickness $(L=R$ as a red line and $L=R / 2$ as a blue line). By looking at the field patterns at these resonances [shown for the $L=R / 2$ case in the insets of Fig. 1(b)], it is clear that the peak at a low frequency corresponds to the electric LSP while the one at a higher frequency is the magnetic LSP. This picture is reinforced by analyzing the vectorial plots also displayed in the same insets ( $E$-field lines for the low-frequency peak and $H$-field lines for the high-frequency peak). In the first case (upper inset), the arrows go from one end of the structure to the other, as corresponds to an electrical dipole, whereas in the lower inset, the arrows show how the magnetic field is circulating around the disk, as in a magnetic dipole. Hence, while subwavelength corrugated infinitely long cylinders present a magnetic LSP that is very close in frequency to the electric LSP, finite corrugated disks support a magnetic LSP at a different frequency than the electric LSP.

Here, it is important to compare the EM modes supported by corrugated metal cylinders or disks with those emerging in subwavelength dielectric particles. These particles also show magnetic and electric dipole modes in the same frequency range [30,31]. The upper right inset of Fig. 1(b) compares the calculated SCS for three different structures: (i) a corrugated PEC disk, as in the main panel; (ii) a dielectric disk of radius $R$ and the same refractive index $n_{g}$; and (iii) a dielectric shell of width $h$ surrounding a PEC core of radius $r$. As opposed to the modes for the corrugated PEC disk, for dielectric particles, the magnetic dipole resonance lies at lower energies than the electric dipole resonance. The physical origin of this opposite frequency ordering for metallic and dielectric disks is related to the dissimilar shapes of the corresponding electric field line patterns. This distinct behavior implies that the corrugated PEC structure cannot be interpreted as an effective isotropic dielectric medium. Rather than that, in the limit $\lambda \gg R$, the region with grooves can be mapped into a metamaterial with both anisotropic permittivity and permeability tensors. Detailed accounts of both the rationale for the different frequency ordering of the modes and the anisotropic metamaterial equivalence are discussed in depth in the Supplemental Material [29].

Although these magnetic LSP modes (and those supported by dielectric particles) are very promising in order to create dipolar magnetic resonances, they rely on the inclusion of a dielectric material with a very high $n_{g}$, which could limit their feasible implementation. Building up magnetic LSP modes supported by purely metallic structures would be much more convenient. Equation (1) gives a clue on how to proceed, as it predicts that, in the subwavelength limit, the positions of the complex resonance frequencies are mainly controlled by the product $n_{g} h$. Therefore, an interesting design is to replace the grooves with $h \leq R$ that are filled with a dielectric material by very long empty grooves that possess an effective length much larger than $R$. Here, we introduce two novel structures that exploit this basic idea: PEC cylinders or disks corrugated with meanders and with spiral grooves. In principle, it is not clear whether the hypothesis that bent grooves behave like straight larger ones is valid, due to possible internal reflections at the bends. However, our numerical results confirm the validity of this approach. Notice here that the bend geometry can also be optimized to compensate for such reflections [32]. In Fig. 2(a), we present detailed results on the LSP modes in PEC structures corrugated with meanders. The geometry we are considering is sketched as an inset: A cylinder of radius $R$ is textured with four meander-shaped grooves of effective depth $h_{m} \approx N(R-r) / 4$. The computed SCS for such a structure is plotted as a black line for the 2D case. Similarly to the already discussed PEC cylinder with filled grooves, the SCS shows a dominant peak that reveals electrical dipole and magnetic dipole field patterns, as shown in Figs. 2(b) and 2(c). A rough estimation based on Eq. (1) (where $h$ is substituted by $h_{m}$ ) predicts spectral locations of these two dipolar modes at $k_{0} R=\pi R /\left(2 h_{m}\right) \approx 0.262$, very close to the numerical value 0.268 . 3D structures corrugated with 

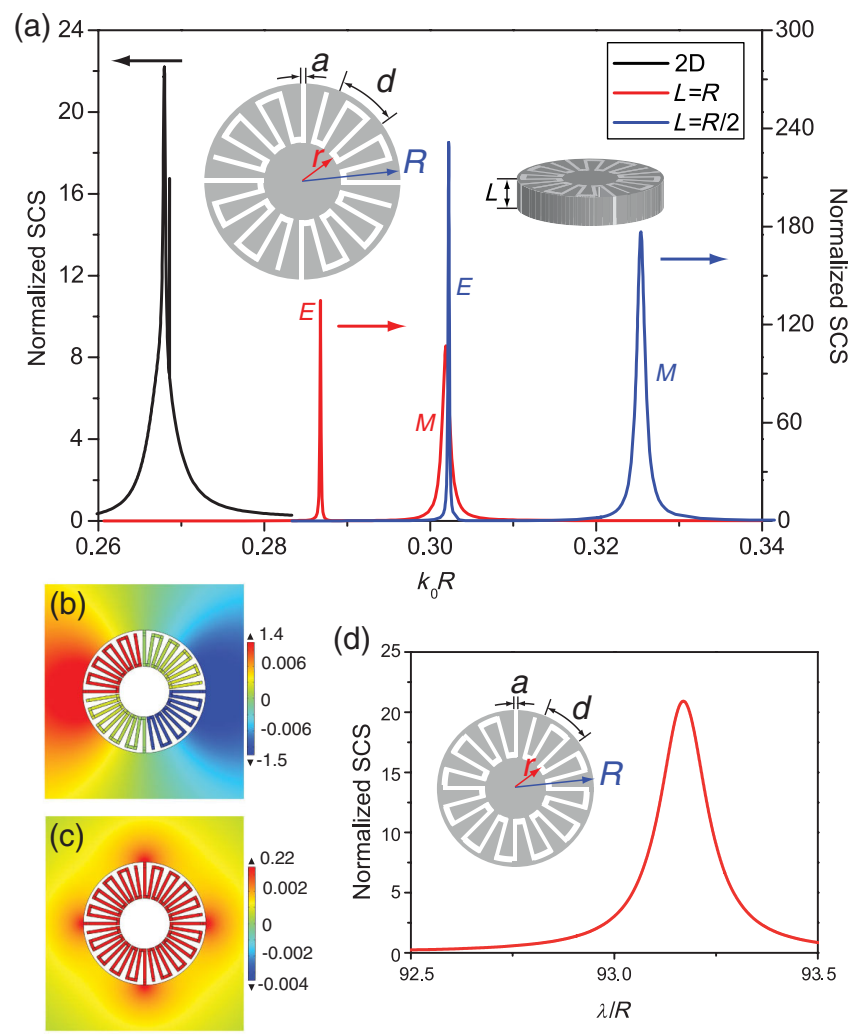

FIG. 2. Magnetic LSPs in PEC subwavelength structures without dielectric filling. (a) Particles corrugated with meanderlike grooves. (The geometry is sketched as an inset.) The plot shows the SCS for an infinite cylinder (black line) and disks of thicknesses $L=R$ (red line) and $L=R / 2$ (blue line), corrugated with four meanders and with parameters $N=40, a=0.3 d$, $n_{g}=1$, and effective depth $h_{m} \approx 6 R$. (b),(c) Field pattern ( $H_{z}$ amplitude) for the $2 \mathrm{D}$ case at the dominant peak in the SCS $k_{0} R=0.268$ showing the (b) electric dipole and (c) magnetic dipole modes, respectively. (d) Normalized SCS for an infinite cylinder with geometrical parameters as in (a) but corrugated with just one meander.

meanders also behave similarly to PEC disks corrugated with filled grooves. In particular, the SCS for disks with meanders ( $L=R$ as a red line and $L=R / 2$ as a blue line) shows a shift in the resonant modes, with the magnetic LSP spectrally located at higher frequencies than the electrical LSP. One of the main assets of our design is that it allows us to create EM modes whose resonant wavelengths can be made much larger than the size of the object. This asset is illustrated in Fig. 2(d), in which the normalized SCS of an infinite cylinder corrugated with only one meander displays a resonance at a wavelength that is around 100 times larger than the radius.

A different way of achieving grooves of very long length is based on a spiral geometry. The structure consists of a $2 \mathrm{D}$ PEC cylinder or a 3D disk of radius $R$ drilled with four spiral-shaped grooves that are wrapped around a small cylinder of radius $r$, as presented in the Supplemental Material [29]. Note that spiral structures with a different geometry have been explored before, both in the microwave and metamaterials areas of research. Spiral structures have been traditionally used as frequency-independent antennas or for polarization control at low frequencies [33-35]. More recently, spiral-based metamaterials like Swiss rolls [15] or one-spiral resonators [36,37] have been introduced in order to improve the magnetic response of split-ring resonators [15]. However, the physical principle of our spiral structure is different, as its design is intended to mimic the EM response of a corrugated cylinder with shallow grooves and filled with a dielectric. An important asset of our design is that these electric and magnetic LSP resonances also exist for ultrathin $(L \ll R)$ metal films, as we will show below. Therefore, meander and spiral structures could be used as building blocks in the design of 2D metamaterials (i.e., metasurfaces) $[38,39]$.

In order to provide an experimental proof-of-principle verification of the magnetic LSP modes, we have fabricated ultrathin metallic spiral structures. For practical reasons, here we work in the microwave regime, but magnetic LSP modes could also be devised at higher frequencies (terahertz up to midinfrared energies) by just a proper down-scaling of the structure analyzed in this experimental study. The fabricated structure consists of four metallic spiral arms [see the geometry sketch in Fig. 3(a) and Supplemental Material [29] Fig. S7a] wrapped 1.5 turns around a small metallic disk of radius $r$ such that the particle has an outer radius $R$. Each strip has width $w$, and two neighboring arms are separated by a distance $d$. The textured copper disks (radius $R=9.5 \mathrm{~mm}$ ) have a thickness that is much smaller than the radius: $L=0.035 \mathrm{~mm}$. The whole ultrathin structure is fabricated on top of a FR4 substrate using the standard printed circuit-board fabrication process. The substrate has a thickness $t_{s}=0.8 \mathrm{~mm}$ and a dielectric permittivity $\epsilon_{s}=3.5$.

In Fig. 3(a), we present the measured near-field spectrum for the ultrathin spiral structure (red line). The EM response of the structure is measured by means of a transmitting monopole antenna that is placed $3 \mathrm{~mm}$ away from one side of the sample to excite the modes and a receiving monopole antenna that is located at the other side of the sample to detect the resonance spectrum, as shown in Supplemental Material Fig. S7b [29]. Both antennas are connected to a vector network analyzer (Agilent N5230C). In the plot, we can clearly observe two distinctive resonance peaks at frequencies $f=1.1 \mathrm{GHz}\left(k_{0} R=0.22\right)$ and $f=1.87 \mathrm{GHz}$ $\left(k_{0} R=0.37\right)$. To reveal the physical nature of these two resonances, we have calculated the extinction cross-section (ECS) spectrum for the same structure [see the black line in Fig. 3(a)]. It shows two resonances whose spectral locations are in excellent agreement with the experimental results. In this case, the ECS, which is obtained as the sum of absorption cross section and radiation cross section, has been calculated by means of full wave simulations [40] in which both the metal absorption and the presence of a dielectric substrate are 


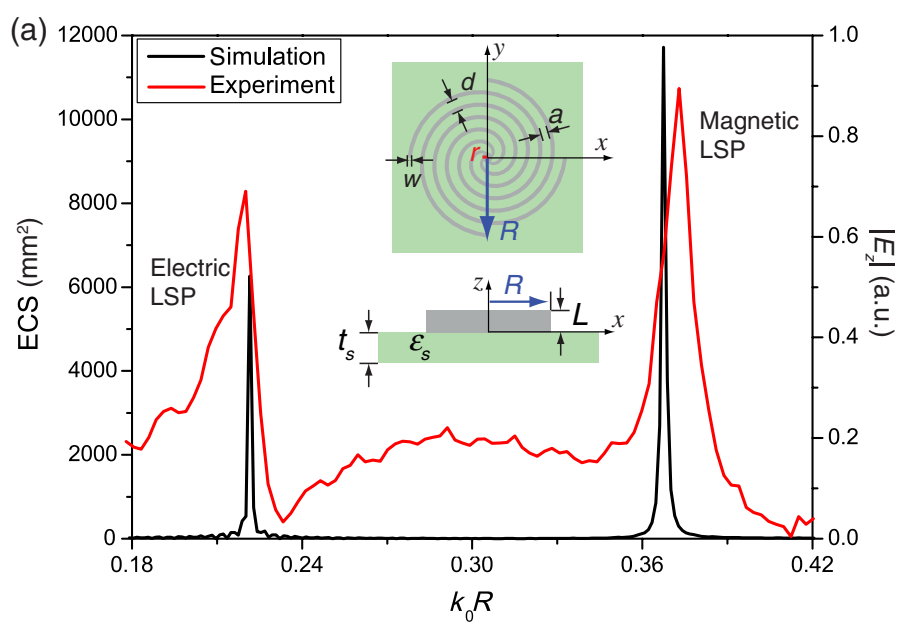

(b)

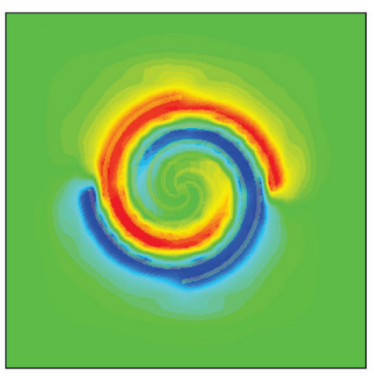

Electric dipole (c)

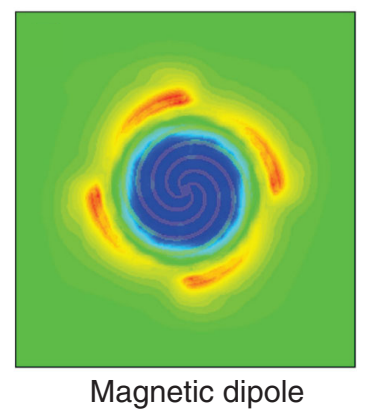

FIG. 3. Resonance spectrum for subwavelength ultrathin textured metallic disks. (a) Experimental near-field spectrum $\left(\left|E_{z}\right|\right.$ is measured) and calculated ECS spectrum. The insets show a sketch of the structure, which is placed on top of a dielectric of thickness $t_{s}=0.8 \mathrm{~mm}$ and $\epsilon_{s}=3.5$. The parameters of the fabricated structure are $R=9.5 \mathrm{~mm}, r=0.6 \mathrm{~mm}, d=1.508 \mathrm{~mm}, a=1.008 \mathrm{~mm}$, and $L=0.035 \mathrm{~mm}$. (b) Near-field distribution of the $E_{z}$ field component at the electric LSP in an $x-y$ plane cut $1.5 \mathrm{~mm}$ above the ultrathin disk. (c) The same for the magnetic LSP. In the color scale, red and blue indicate positive and negative values, respectively.

fully taken into account. The simulated field patterns at the two resonances are plotted in Figs. 3(b) and 3(c). Similarly to the behavior previously discussed for PEC disks corrugated with meanders and spirals, these plots show that the first resonance is due to the electrical LSP [Fig. 3(b)], while the second peak emerges from the magnetic LSP [Fig. 3(c)].

Furthermore, to demonstrate experimentally the predicted electric and magnetic resonance field patterns, we present in Fig. 4 the measured near-field distributions on the spiral's structure. By means of a near-field scanning technique [41], we have mapped the local (i) transient fields, (ii) phases, and (iii) magnitudes of the $z$ component

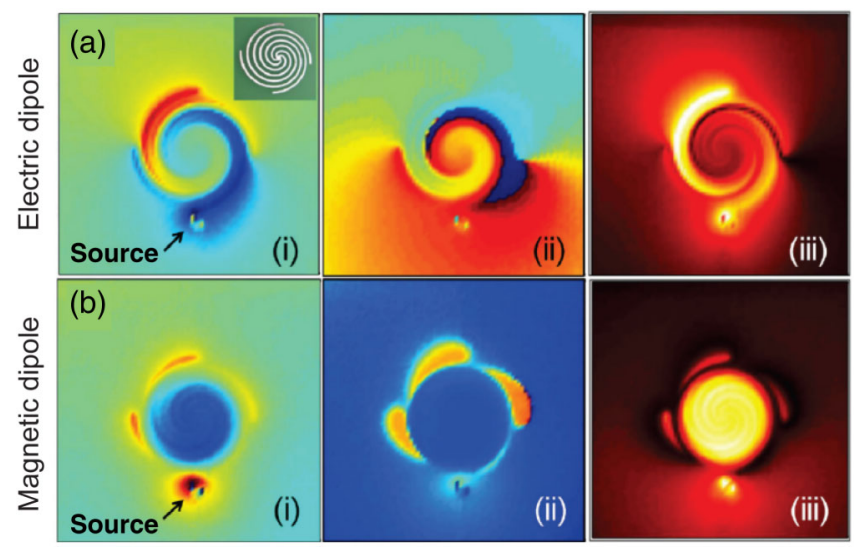

FIG. 4. Measured near-field distribution of the LSP. Measured near-field distributions of (a) the electric dipole LSP at $1.1 \mathrm{GHz}$ and (b) the magnetic dipole LSP at $1.87 \mathrm{GHz}$ for the ultrathin metallic structure. Panels (i), (ii), and (iii) show the transient fields, phases, and magnitudes, respectively. The inset of panel (i) of (a) shows a photograph of the fabricated sample. of the electric field for both the electric and magnetic dipole LSP resonances [Figs. 4(a) and 4(b), respectively]. By comparing both panels (i) of Figs. 4(a) and 4(b) to the numerical results shown in Figs. 3(b) and 3(c), we conclude that the agreement between the simulation results and the experimental images is remarkable. For the electric LSP mode [Fig. 4(a)], we notice in panel (i) that two equivalent electric currents propagate along two curved routes on the metallic surface with opposite directions, resembling an electric dipole along a curved line. Panel (ii) of Fig. 4(a) reveals a dipolarlike phase change of the field, and panel (iii) shows that the field is maximum in the outer part of the spirals. On the other hand, for the magnetic dipole mode [Fig. 4(b)], we can see in panel (i) that the circulating $E_{z}$ field varies between negative and positive, which is analogous to the molecular current that generates the molecular magnetic momentum of Ampere's hypothesis. Panels (ii) and (iii) of Fig. 4(b) show in this case that the phase is uniform in the azimuthal direction while the field is maximum in the center of the structure.

The concept of magnetic localized surface plasmons developed and experimentally verified in this work adds the important ingredient of magnetism to the field of particle plasmonics. The extreme light confinement and high EM fields inherent to localized plasmons combined with a resonant magnetic response open up the possibility of fundamental studies of magnetism at a very deep subwavelength scale. We envisage that our findings could also have important practical implications not only in the rf or terahertz ranges of the EM spectrum but also at infrared energies in which spectroscopic tools are essential in many technologies devoted to the analysis of material properties. 
This work was supported in part by the European Research Council (ERC-2011-AdG proposal No. 290981) and the Spanish government (Grants No. CSD2007-0046Nanolight.es and No. MAT2011-28581-C02). We also acknowledge financial support by the National Science Foundation of China (Grants No. 60990320, No. 60990321, No. 60990324, No. 61171024, No. 61171026, and No. 61138001), by the National High Tech (863) Projects (No. 2012AA030402 and No. 2011AA010202), and by the 111 Project (No. 111-205). P. A. H. and X. S. contributed equally to this work.

[1] W. L. Barnes, A. Dereux, and T. W. Ebbesen, Surface Plasmon Subwavelength Optics, Nature (London) 424, 824 (2003).

[2] S. A. Maier, Plasmonics: Fundamentals and Applications (Springer, New York, 2007).

[3] E. Ozbay, Plasmonics: Merging Photonics and Electronics at Nanoscale Dimensions, Science 311, 189 (2006).

[4] N. J. Halas, S. Lal, W. S. Chang, S. Link, and P. Nordlander, Plasmons in Strongly Coupled Metallic Nanostructures, Chem. Rev. 111, 3913 (2011).

[5] B. A. Munk, Frequency Selective Surfaces: Theory and Design (Wiley-Interscience, New York, 2000).

[6] R. Collin, Field Theory of Guided Waves (IEEE, New York, 1991).

[7] J. B. Pendry, L. Martin-Moreno, and F. J. Garcia-Vidal, Mimicking Surface Plasmons with Structured Surfaces, Science 305, 847 (2004).

[8] A. P. Hibbins, B. R. Evans, and J. R. Sambles, Experimental Verification of Designer Surface Plasmons, Science 308, 670 (2005).

[9] F. J. G. de Abajo and J. J. Sáenz, Electromagnetic Surface Modes in Structured Perfect-Conductor Surfaces, Phys. Rev. Lett. 95, 233901 (2005).

[10] S. A. Maier, S. R. Andrews, L. Martín-Moreno, and F. J. García-Vidal, Terahertz Surface Plasmon-Polariton Propagation and Focusing on Periodically Corrugated Metal Wires, Phys. Rev. Lett. 97, 176805 (2006).

[11] C. R. Williams, S. R. Andrews, S. A. Maier, A. I. Fernández-Domínguez, L. Martín-Moreno, and F. J. García-Vidal, Highly Confined Guiding of Terahertz Surface Plasmon Polaritons on Structured Metal Surfaces, Nat. Photonics 2 , 175 (2008).

[12] N. Yu, Q. J. Wang, M. A. Kats, J. A. Fan, S. P. Khanna, L. Li, A. G. Davies, E. H. Linfield, and F. Capasso, Designer Spoof Surface Plasmon Structures Collimate Terahertz Laser Beams, Nat. Mater. 9, 730 (2010).

[13] M. A. Kats, D. Woolf, R. Blanchard, N. Yu, and F. Capasso, Spoof Plasmon Analogue of Metal-Insulator-Metal Waveguides, Opt. Express 19, 14860 (2011).

[14] E. M. G. Brock, E. Hendry, and A. P. Hibbins, Subwavelength Lateral Confinement of Microwave Surface Waves, Appl. Phys. Lett. 99, 051108 (2011).

[15] J. B. Pendry, A. J. Holden, D. J. Robbins, and W. J. Stewart, Magnetism from Conductors and Enhanced Non Linear Phenomena, IEEE Trans. Microwave Theory Tech. 47, 2075 (1999).
[16] R. A. Shelby, D. R. Smith, and S. Shultz, Experimental Verification of a Negative Index of Refraction, Science 292, 77 (2001).

[17] S. Linden, C. Enkrich, M. Wegener, J. Zhou, T. Koschny, and C. M. Soukoulis, Magnetic Response of Metamaterials at 100 Terahertz, Science 306, 1351 (2004).

[18] J. B. Pendry and D. R. Smith, Reversing Light with Negative Refraction, Phys. Today 57, No. 637 (2004).

[19] C. Enkrich, M. Wegener, S. Linden, S. Burger, L. Zschiedrich, F. Schmidt, J. F. Zhou, T. Koschny, and C. M. Soukoulis, Magnetic Metamaterials at Telecommunication and Visible Frequencies, Phys. Rev. Lett. 95, 203901 (2005).

[20] S. Zhang, W. Fan, B. K. Minhas, A. Frauenglass, K. J. Malloy, and S. R. J. Brueck, Mid-infrared Resonant Magnetic Nanostructures Exhibiting a Negative Permeability, Phys. Rev. Lett. 94, 037402 (2005).

[21] V. M. Shalaev, Optical Negative-Index Metamaterials, Nat. Photonics 1, 41 (2007).

[22] C. M. Soukoulis and M. Wegener, Past Achievements and Future Challenges in the Development of Three-Dimensional Photonic Metamaterials, Nat. Photonics 5, 523 (2011).

[23] J. N. Gollub, D. R. Smith, D. C. Vier, T. Perram, and J. J. Mock, Experimental Characterization of Magnetic Surface Plasmons on Metamaterials with Negative Permeability, Phys. Rev. B 71, 195402 (2005).

[24] A. Pors, E. Moreno, L. Martin-Moreno, J. B. Pendry, and F. J. Garcia-Vidal, Localized Spoof Plasmons Arise While Texturing Closed Surfaces, Phys. Rev. Lett. 108, 223905 (2012).

[25] P. Kildal, Artificially Soft and Hard Surfaces in Electromagnetics, IEEE Trans. Antennas Propag. 38, 1537 (1990).

[26] COMSOL, Multiphysics 4.2a, http://www.comsol.com.

[27] C. F. Bohren and D. R. Huffman, Absorption and Scattering of Light by Small Particles (Wiley, New York, 1983).

[28] For a review paper, see A. F. Harvey, Periodic and Guiding Structures at Microwave Frequencies, IRE Trans. Microwave Theory Tech. 8, 30 (1960).

[29] See Supplemental Material at http://link.aps.org/ supplemental/10.1103/PhysRevX.4.021003 for details on the analytical calculation, the frequency ordering of the resonances, the metamaterial approximation, the spiralshaped corrugated disks, and the fabricated sample and experimental measurement.

[30] A. Garcia-Etxarri, R. Gomez-Medina, L. S. Froufe-Perez, C. Lopez, L. Chantada, F. Scheffold, J. Aizpurua, M. NietoVesperinas, and J. J. Saenz, Strong Magnetic Response of Submicron Silicon Particles in the Infrared, Opt. Express 19, 4815 (2011).

[31] A. I. Kuznetsov, A. E. Miroshnichenko, Y. H. Fu, J. B. Zhang, and B. Lukyanchuk, Magnetic Light, Sci. Rep. 2, 492 (2012).

[32] F. Alessandri, M. Mongiardo, and R. Sorrentino, Rigorous Mode Matching Analysis of Mitered E-Plane Bends in Rectangular Waveguide, IEEE Microwave Guided Wave Lett. 4, 408 (1994).

[33] J. Dyson, The Equiangular Spiral Antenna, IEEE Trans. Antennas Propag. 7, 181 (1959).

[34] J. A. Kaiser, The Archimedean Two Wire Spiral Antenna, IEEE Trans. Antennas Propag. 8, 312 (1960).

[35] C. A. Balanis, Antenna Theory-Analysis and Design (Wiley, New York, 2005). 
[36] J. D. Baena, R. Marqués, F. Medina, and J. Martel, Artificial Magnetic Metamaterial Design by Using Spiral Resonators, Phys. Rev. B 69, 014402 (2004).

[37] F. Bilotti, A. Toscano, and L. Vegni, Design of Spiral and Multiple Split-Ring Resonators for the Realization of Miniaturized Metamaterial Samples, IEEE Trans. Antennas Propag. 55, 2258 (2007).

[38] N. Yu, P. Genevet, M. A. Kats, F. Aieta, J.-P. Tetienne, F. Capasso, and Z. Gaburro, Light Propagation with Phase
Discontinuities: Generalized Laws of Reflection and Refraction, Science 334, 333 (2011).

[39] A. V. Kildishev, A. Boltasseva, and V. M. Shalaev, Planar Photonics with Metasurfaces, Science 339, 1232009 (2013).

[40] CST, Microwave Studio, http://www.cst.com.

[41] H.F. Ma and T. J. Cui, Three-Dimensional Broadband Ground-Plane Cloak Made of Metamaterials, Nat. Commun. 1, 21 (2010). 